\title{
Distributed Models for Brokerage on Electronic Commerce ${ }^{1}$
}

\author{
Isabel Gallego, isabel@ac.upc.es \\ Jaime Delgado, delgado@ac.upc.es \\ Josê J. Acebrón, acebron@ac.upc.es \\ Telematic Applications Group \\ Computer Architecture Department \\ Universitat Politècnica de Catalunya (UPC) \\ Barcelona, Spain
}

\begin{abstract}
With the development of Electronic Commerce (EC), the need of offering brokerage facilities in a distributed environment has arisen. Customers need, in many situations, systems (broker agents) that simplify them the access to the different products and services provided by suppliers. On the other hand, the existence of brokers increases the globality of $\mathrm{EC}$, since customers are even more unaware of the location of their final goods providers.

To help on this, several brokerage systems are being developed in different contexts. However, how to organise broker agents in a distributed EC environment is not always being approached in the same way. This paper concentrates on this problem, and considers the different work in the field currently going on. A special focus is made on European research projects.

This paper proposes several distributed models for Electronic Commerce, focussing on the case when brokerage facilities are included. In particular, we are proposing different models, i.e. a functional model, and operational model and an architectural model. In order to show the feasibility of the models, how they are applied to a specific implementation is shown.

Keywords: Electronic Commerce, Electronic Brokerage, Customer, Supplier, and Distributed Models.
\end{abstract}

\section{Introduction}

Electronic brokerage systems is one of the areas inside electronic commerce, where distributed systems may play a key role. This paper presents several distributed models for brokerage on electronic commerce where these ideas are illustrated.

This document is structured in five parts. The first one is a proposal for a functional model for an electronic brokerage system. The second one explains an operational model where a series of phases have been identified. These phases are needed for a customer to obtain a product or service from a supplier through a broker agent. For every phase, a list of associated functions is also identified.

The third part of the paper explains the architectural model for the three levels identified in the functional model: customer, supplier and broker level.

The fourth part describes two scenarios where the two previous models can be applied; the first scenario refers to the buying of an electronic product (delivered then

${ }^{1}$ This work has been partly supported by the European Commission (ACTS096) and the Spanish Administration (TIC95-0903-C02-01). 
through the network), and the second one describes how to order and buy an electronic service (produced off-line). Finally, the last part describes how the different proposed models are applied to a specific real implementation, that of the ACTS MULTIMEDIATOR (Multimedia Publishing Brokerage System) project [1], that is intended for the publishing industry.

The European Commission is supporting $R \& D$ activities in the area of electronic commerce inside different programmes, such as ACTS [1]. The authors of this paper have participated in EU co-funded projects and contributed to produce guidelines for electronic commerce [2], [3], [4].

There are also standardisation and specification efforts in the area of electronic commerce. Examples are CEN/ISSS (previously EWOS) [5], EBES [6] and industrial fora, like OMG [7]. All of them are specifying models and systems for electronic commerce.

\section{Functional Model}

As illustrated in Figure 1, several levels can be distinguished in a functional model for electronic brokerage:

- Customer level: these are the users of the service.

- Broker level: in this level, one electronic broker or several electronic brokers provide the service requested by the customer, using the services offered by the suppliers.

- Supplier level: suppliers offer their services to one or more brokers.

- Service level: electronic brokerage requires additional services that are not specific of it, such as security and electronic payment.

- Distribution level: the result of the service should be distributed from the suppliers to the customers: the distribution can be electronic (in the case of electronic information) or physical, in the general case.

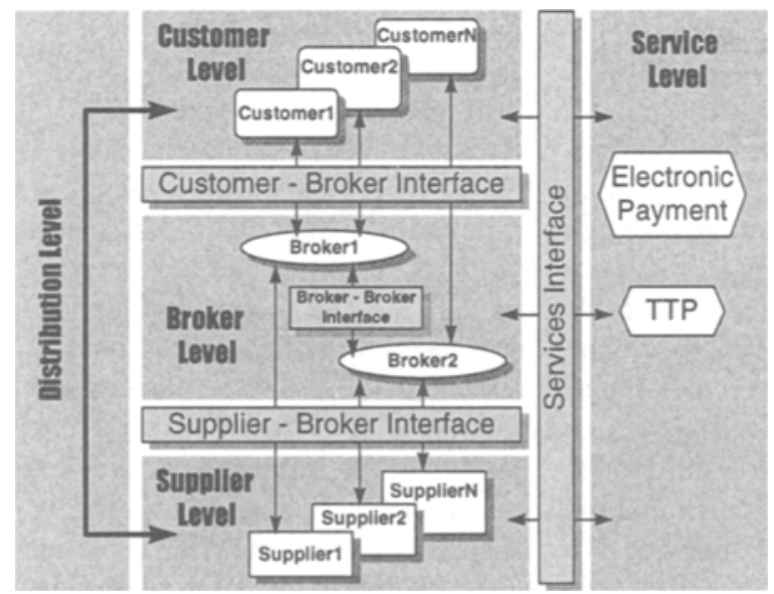

Fig. 1. Functional model for an electronic brokerage system 
Figure 1 also illustrates the interfaces between the different modules, which play a key role in the interoperability between them. The following interfaces are identified:

- Customer-Broker: this interface specifies the interaction between the customer level and the brokerage level. This interface allows to present the broker level and the supplier level as a global service level to the customer, who does not need to deal with the location and characteristics of the suppliers.

- Supplier-Broker: this interface specifies the interaction between the supplier and the broker level.

- Broker-Broker: this interface defines the interaction between different brokers that co-operate in order to improve their services. This is a key point in the provision of a distributed brokerage service. Where several broker systems can co-operate to provide a more "global" service.

- Services: the customer, supplier and broker levels require additional services in order to interwork correctly. This interface specifies the interaction of these levels with the supporting services, such as security or payment.

\section{Operational Model}

The operations related to an electronic brokerage system can be categorised into two types:

- Service-oriented: those that occur during the provision of services to the customers;

- System-oriented: those that provide the users with some added-value system operations.

We can identify the phases that are carried out generally when a customer requests a product or service to a supplier through an electronic brokerage system.

In the case of the service-oriented phases, we can identify the following sub-phases:

- Service Identification: In this sub-phase, the final user searches and/or asks for services available in the broker system. The user makes a selection in order to identify those services or products that fulfil his interest.

- Service Request: Once the service or product has been selected, the user must perform the formal ordering for the acquisition, with which both parts, seller and buyer, become responsible to fulfil the conditions and statements of an established contract. All the elements that take part in this process need to identify themselves using a reliable authentication mechanism.

- Agreement: If the user has bought a final product that can be delivered in an electronic way, the supplier performs the delivery of the product and the user issues the payment (or takes the responsibility for the future payment). If the item requested was a service, the conditions for the development of the service (service parameters, delivery specifications, etc.) are to be agreed in this point.

- Post-agreement: When the user requests for a customised service, it is necessary to specify a procedure (after the agreement) that allows the follow-up of the service until the final delivery. In this phase, the user can check if the service is being carried out as agreed. If this phase takes place, the payment (whole or partial) may be post-poned. There may be a temporal overlapping between the agreement and the post-agreement phases.

In the case of the system-oriented phases, the following sub-phases can be identified: 
- Maintenance: It allows the users to access some maintenance services such as automatic software upgrading from the broker.

- Service-Update: The suppliers should be also able to update the information that the broker maintains about them. It should allow to update catalogues and/or to add new offers or services.

- Contract re-negotiation: It allows the customers and suppliers that may have some contractual relationship with the broker, to modify the contract previously established (e.g. to modify the payment conditions).

The next clauses present some aspects of these phases and sub-phases in more detail.

\subsection{Service-Oriented Phases}

The following sub-clauses present some concrete aspects of the service-oriented phases described above.

Service Identification. The following list describes some of the most relevant actions associated to the service identification phase. They are mainly based on [2].

SEARCH The customer can buy products and services. This action is responsible for searching and identifying information about products and services that fit a user description.

BROWSER The broker agent can provide a form to the users for queries or job requests. This functionality is carried out through the forms mechanism based, for example, in WWW technology.

METADATA The information about what the different suppliers are able to provide is stored in the broker agent as metadata in a database.

PROFILE It is possible to define user profiles with information like access rights, supported data formats, etc. This information is stored in a management database.

CATALOGUE Customers can obtain information about suppliers and services with brochures and forms (for subscription). This information is stored in the broker database.

Other actions identified in this phase are (see [4]): locate, agency, customer and directory services.

Service Request. The following list describes some of the most relevant actions associated to the service request phase. They are mainly based on [2].

CONTRACT It is possible to manage contracts with the contract management module, providing collaborative contract construction, negotiation, execution, signatures and service plan. The associated information of the contract is stored in the broker management database.

Other actions identified in this phase are (see [4]): brokerage and selection.

Agreement. The following list describes some of the most relevant actions associated to the agreement phase. They are mainly based on [2].

DELIVERY

Manages the delivery of items to the customer. It can support two kinds of delivery, electronic delivery and physical delivery. In some cases, such as in the multimedia 
information services, it is interesting to provide the possibility to convert the information delivered by the supplier to the format selected by the user.

PAYMENT Provides a mechanism for doing and monitoring payment, that can be off-line or on-line; in the second case the billing module is involved and this information is stored in the broker management database.

AUTHENTICATION Provides a mechanism, which allows users to guarantee their identity to a brokerage system by an authentication process.

CERTIFICATION Supports users identity checking and certification by a certificate.

FEEDBACK

Allows the users to have feedback on a particular action, transaction, and network or system failures. Users can obtain feedback information of the process in course through the broker management database.

Other actions identified in this phase are (see [4]): order and service management.

Post-Agreement. The following list describes some of the most relevant actions associated to the post-agreement phase. This is one of the main contributions, detailed in [3] and [4], of this paper to [2].

CUSTOM

SERVICES

SERVICE

STATUS

CUSTOMER

UPLOAD

SERVICE POOL This management function allows the broker agent to queue the different pending requests associated to the services, this information is stored in the broker management database.

AFTER

AGREEMENT

This functionality provides the customers with the possibility of obtaining tailored services using, for example, WWW-based technology by filling forms.

The customers can know the status of all their pending requests. The information is stored in the broker management database.

This functionality provides the customers with the possibility to send information to the suppliers, and store it, temporarily, in the broker agent database, e.g. uploading a source document for its translation.

Allows users to buy tailored agreed services and to control the operation until the final delivery of the service. When a customer buys a custom service, it is necessary to provide him with functionality to follow up the process until the final delivery.

\subsection{System-Oriented Phases}

As system-oriented phases, we understand those functionality that can be carried out at any time and independently of the provision of services, and are intended for the maintenance of the system. 
Maintenance.

SOFTWARE UPDATING

Service Update.

SERVICE

UPDATING
This functionality allows the users to update the software necessary to access to the broker system.

This functionality allows the suppliers to update their offer: new catalogues, discounts, price reductions, etc.

\section{Contract Re-negotiation.}

CONTRACT This functionality allows customers and suppliers to reRENEGOTIATION negotiate the conditions of their contracts with the electronic broker.

\section{Architectural Model}

As described in the functional model, the following main levels can be distinguished in an electronic brokerage system:

- Customer level

- Brokerage level

- Supplier level

For each level, several modules, which are responsible for performing the operations described above, can be identified.

In the customer level, the final users can interact with the electronic brokerage system in two different ways:

- Direct Access: if the user previously knows the product to order, the access can be done directly without any browsing or searching.

- Navigation Access: if the user does not know previously the product or service to be ordered. This mechanism allows to browse and search in the broker information.

In the supplier level, the modules are similar to those of the customer, but there is an additional module to allow the broker (or brokers) to access its local information (detailed information and final products). They are:

- Direct Access: Similar to the customer level, but with more facilities, including the updating of the broker database with the information of every supplier.

- Navigation Access: Similar to the customer level, but with more facilities, including the updating of the forms stored in the broker with the information about the available services and products of every supplier.

- Supplier Database: allows a broker (or brokers) to access information stored in the supplier system.

The broker level is the most complex system, the following modules are identified:

- Direct Access Handler: This module manages the direct access to the broker services.

- Navigation Access Handler: Allows the users to navigate and browse in the broker information about services and products. 
- General \& Management Database: This module manages the Database of the broker which contains management information (e.g. accounting information), users information (e.g. user profiles) and product and services information.

- Supporting Services: This module provides services for electronic brokerage that are required as supporting services, but are not part of electronic brokerage. Some of these services are payment, security services, electronic copyright management, etc.

- Search Module: This module provides specific search functionality to assist the users of the system. The search functionality may integrate some intelligent behaviour such as multi-lingual searching.

Figure 2 shows the architectural model of the systems involved in electronic brokerage, including the modules described above.

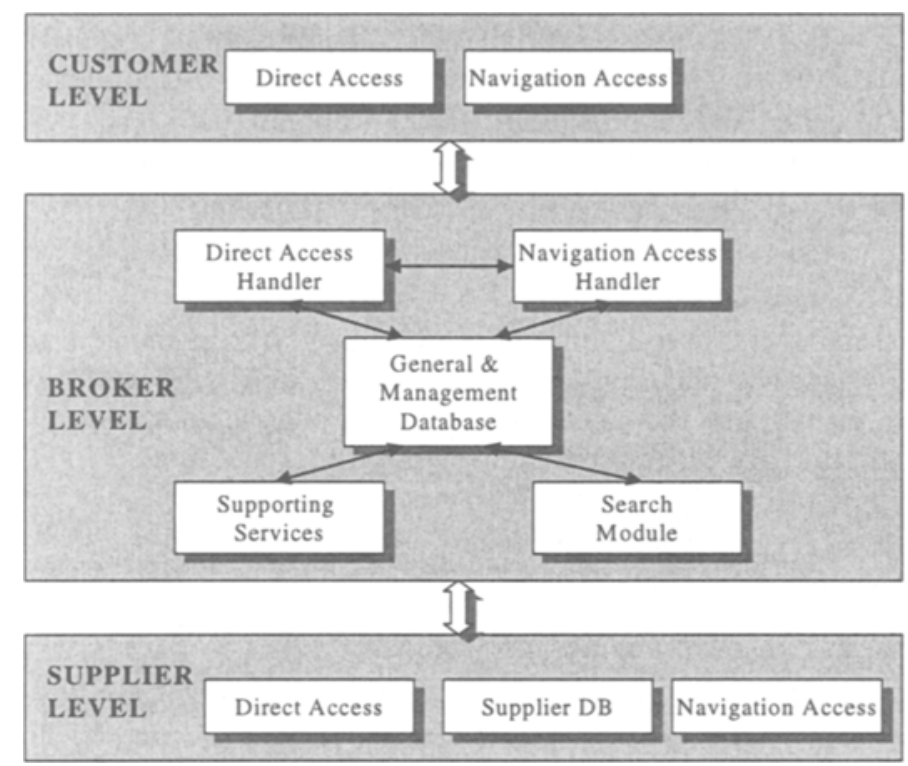

Fig. 2. General architectural model for an electronic brokerage system

The modules of the broker level can be distributed among different systems in order to provide a more powerful service.

\subsection{Mapping of the Functional Model onto the Architectural Model}

We can relate the two described models by making a mapping of the operational model onto the architectural model. The mapping identifies which operation (or functionality) is to be provided by each module described in the architectural model. This mapping is shown in the following table. 


\begin{tabular}{|l|l|}
\hline \multicolumn{1}{|c|}{ Module } & \multicolumn{1}{c|}{ Functions } \\
\hline Direct Access & $\begin{array}{l}\text { Profile, metadata, catalogue, directory services, customer, } \\
\text { brokerage, contract, item and stream delivery, feedback, } \\
\text { service management, service status, service pool, custom } \\
\text { services, customer upload. }\end{array}$ \\
\hline Navigation & $\begin{array}{l}\text { Customer, agency, search, locate, browser, selection, after } \\
\text { agreement and order. }\end{array}$ \\
\hline Supporting Services & $\begin{array}{l}\text { Payment, certification, authentication, software \& service } \\
\text { updating, format conversion and contract re-negotiation. }\end{array}$ \\
\hline
\end{tabular}

\section{Scenarios}

This clause provides a description of two scenarios where the ideas presented above are reflected. The phases carried out during the process are identified and illustrated in real-life scenarios. The first scenario presents a typical service that can be provided over electronic brokerage systems, which is selling of electronic images. It corresponds to the commerce of a specific and existing product. The second scenario presents a customised service of video composition. This scenario illustrates the problems that arise in the provision of a customised service over electronic brokerage systems.

\subsection{On-line Image Shopping}

In this scenario, the end-user is someone who wants to buy an image and will use an electronic system. The end-users can select images in a catalogue offered by the brokerage level and get additional information about the images like price, size, format, etc. In this case the end-users buy an electronic end-product and can obtain it automatically by electronic delivery. The brokerage level (which may consist of one or several brokerage systems) will act as intermediary between many end-users (customers) and many suppliers.

Firstly, in the service identification phase, the user access to the electronic brokerage level using the customer functionality, all functionalities are described in section 3 , and are presented in italics for easy reference. The brokerage level uses the information about the customer (profile, agency) to facilitate the locating and searching of the image catalogues (directory services, metadata).

In the service request phase, the user decides which image/s to buy (brokerage, selection) and proceeds with the negotiation of the buying contract (contract).

In the agreement phase, the customer orders the image (order) and the supplier delivers the electronic image (delivery). The customer pays the image. In order to provide for a reliable payment, the parties involved need to be authenticated (authentication and certification). The user is able to follow-up the process (service management and feedback).

There is no post-agreement phase in this scenario, since the customer orders a predefined, already existing, product.

Figure 3 illustrates the steps in the provision of on-line image shopping over the electronic brokerage level. 


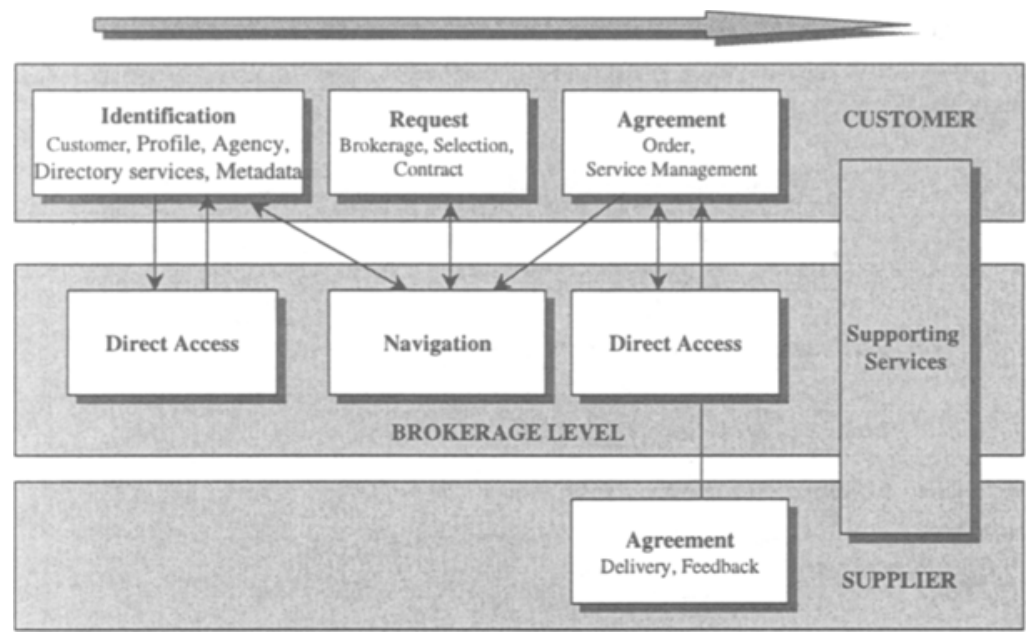

Fig. 3. Steps in the provision of on-line image shopping over an electronic broker

\subsection{Customised Video Composition}

In this scenario, a customer can order the creation of a video resulting from the composition of a set of video elements (MPEG4 video objects, for example) that have been selected by the customer in some video object catalogues. In the selection process, the customer can select the objects that will be integrated in the video, and their characteristics (placement, repetition, etc.). The supplier will compose then a video sequence using a professional MPEG4 composition tool and will deliver the resulting video to the customer. This is a scenario where the customer buys a service, whose results will be delivered latter, after the realisation of the service.

Firstly, the customer browses the catalogues of video services offered by the brokerage level (service identification phase). Once the service has been selected (selection), customer and supplier negotiate the contract (contract) and the video is ordered. In this case, the elaboration of a video is requested, and some aspects, such as the delivery time, are to be agreed (service request phase). This is one of the main differences between this scenario and the previous one.

In this scenario, the agreement and post-agreement phases are overlapped in time; for example, the payment is postponed until the final video delivery.

After the ordering of the service (order), in the post-agreement phase, the users (customers and suppliers) can perform the customisation of the service (custom services). The customers can also know the status of the pending services (service status, service pool). This is another relevant difference with the previous scenario.

Once the supplier has delivered the final video product (delivery), the user proceeds to the payment as agreed in the contract (payment). The customer can follow up the development of the service (service management, feedback). For a guaranteed payment, the users need to be authenticated and the transactions are to be carried out in a secure way.

The final video may be in MPEG4 format (if the customer is able to process it) or it can be converted to any other video format, such as MPEG1 or MPEG2 (delivery). In 
this case, the delivery action includes a customisation of the information format depending of the characteristics of the user.

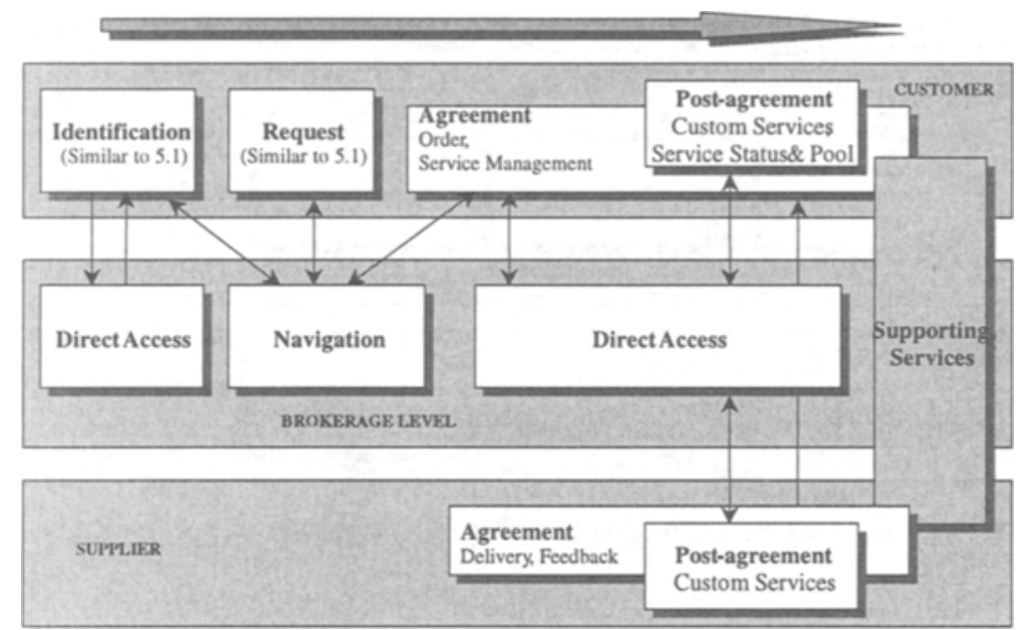

Fig. 4. Steps in the provision of customised video composition over an electronic broker

Figure 4 illustrates the steps in the provision of customised video composition over an electronic broker.

\section{Validation of the Models}

The models presented in this paper have been validated in a real case, the ACTS MULTIMEDIATOR (Multimedia Publishing Brokerage Service) project, that is developing a multimedia brokerage application, as a first step of more complex Electronic Commerce applications. The key target of the project is multimedia publishing industry, where multimedia publications developers, the Customers, may need to buy products and services, from the Suppliers, to produce their electronic publications. A basic model for brokerage has been implemented, allowing Customers to easily negotiate with a Broker Machine (BM) the multimedia product or service they want from the Suppliers, who, in turn, receive orders from the BM and deliver products and services to it. For this purpose, a database containing information on products and services to sell is included in the BM. Suppliers are responsible for keeping up to date the information on that database. In this case the brokerage service is provided by only one system and therefore not distributed.

In MULTIMEDIATOR, the communication is always between the users' site and the $\mathrm{BM}$ (there is not direct communication of a given user with other users). Customers connect to the BM when they want to look for (and, eventually, buy) products or services. On the other hand, the BM accesses suppliers when a product or service has been bought, or they connect to the BM when they want to update the information about their offers. Three access mechanisms are provided: WWW-based forms, Interactive (whiteboard-based), and DFR (Document Filing and Retrieval) based. In 
the interactive access, there is an interaction between customer and supplier, but through the broker machine.

The project started in September 1995, and has finished in March 1998. The results include prototypes for the customer and supplier systems and the broker machine, apart from a model, architecture, and several contributions to standardisation. During the last months, several international ATM trials took place, where the system was tested in a real environment. Some partners in the consortium have serious plans for exploitation of the results. More information can be found in [8] and [9].

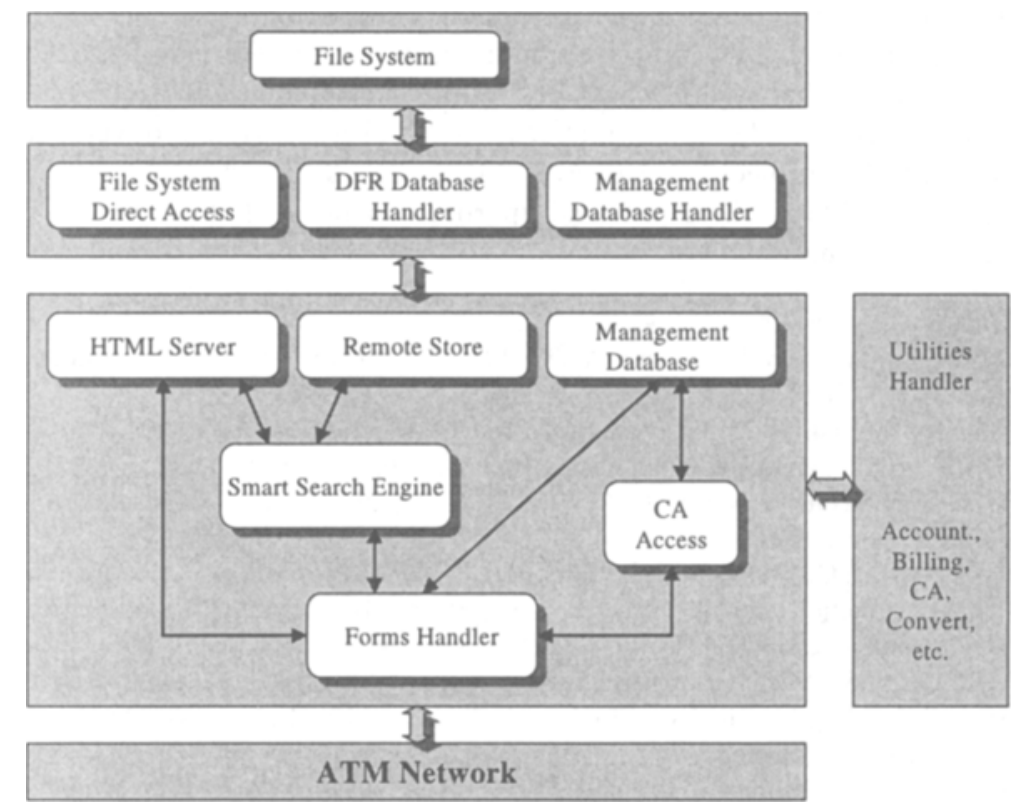

Fig. 5. MULTIMEDIATOR architectural model

The MULTIMEDIATOR broker machine has its own architecture that is shown in figure 5. It can be seen from the picture, that, if comparing to the architectural model proposed in section 4, MULTIMEDIATOR follows that model. The mapping of the five architectural blocks specified in section 4 (Direct Access Handler, Navigation Access Handler, General \& Management Database, Supporting Services and Search Module) to the MULTIMEDIATOR blocks in figure 5 is shown in the following table.

\begin{tabular}{|l|l|}
\hline \multicolumn{1}{|c|}{ Architectural model } & \multicolumn{1}{c|}{ MULTIMEDIATOR blocks } \\
\hline Direct Access Handler & Remote Store Handler \\
\hline Navigation Access Handler & Forms Handler \\
\hline General \& Management Database & DFR Database, Management Database \\
\hline Supporting Services & Utilities, Certification Authority \\
\hline Search Module & Smart Search Engine \\
\hline
\end{tabular}




\section{Conclusions}

The paper has presented several distributed models for electronic brokerage applications, as a part of Electronic Commerce applications, from three different points of view:

- Functional model.

- Operational model.

- Architectural model.

The three points of view are complementary and co-ordinated between them.

Existing work in the field has been considered, and the work presented here has been contributed to the development of a Guideline on "Models of electronic brokerage" [2] [3] [4], initiated by some research projects of the ACTS (Advanced Communications, Technologies \& Services) European Commission programme [1].

The ideas have been validated in a real case, the brokerage system developed in the MULTIMEDIATOR project [9]. However, the models do not pretend to be the only solution to the problem, but a practical approach to most of the cases in which a brokerage system is needed.

\section{References}

1. European Commission Document AC971392-PS: "ACTS (Advanced Communications Technologies \& Services) 97, Project Summaries", June 1997.

2. ACTS SIA Guideline 3 "Enterprise Models of Electronic Brokerage" Edited by Dr. Stephen Plagemann and Dr. Jenny Hands. Version 1.6, September 1997.

3. MULTIMEDIATOR Document A0096/UPC/DAC/PI/I/031/a1 "Contribution to SIA Guideline 3", May 1997.

4. MULTIMEDIATOR Document A0096/UPC/DAC/PI/I/038/a1 "Application and Middleware functions for electronic brokerage - A MULTIMEDIATOR contribution to SIA-G3", September 1997.

5. EWOS/ETG 066: "EWOS Technical guide on electronic commerce", September 1996.

6. EBES "Building Blocks for Electronic Commerce", October 1997. http://www.cenorm.be/ebes/

7. OMG Electronic Commerce Domain Task Force "EC-DTF Reference Model (Draft)", http://www.osm.net/ec-dtf/working.html

8. Martí R. and Delgado J. "Use of WWW technology for Client/Server Brokerage Applications in MULTIMEDIATOR”, IS\&N'97, Springer Ed. May 1997.

9. MULTIMEDIATOR Web Page, http://www.ac.upc.es/multimediator/ 